\title{
Freezing a quantum magnet by repeated quantum interference: An experimental realization
}

\author{
Swathi S. Hegde, ${ }^{*}$ Hemant Katiyar, ${ }^{*}$ and T. S. Mahesh ${ }^{\dagger}$ \\ Department of Physics and NMR Research Center, Indian Institute of Science Education and Research, Pune 411008, India
}

\author{
Arnab Das ${ }^{\ddagger}$ \\ Theoretical Physics Department, Indian Association for the Cultivation of Science, Kolkata 700032, India \\ and Max-Planck-Institut für Physik komplexer Systeme, 01187 Dresden, Germany \\ (Received 21 August 2013; revised manuscript received 17 September 2014; published 7 November 2014)
}

\begin{abstract}
We experimentally demonstrate the phenomenon of dynamical many-body freezing in a periodically driven Ising chain. Theoretically [A. Das, Phys. Rev. B 82, 172402 (2010)], for certain values of the drive parameters all fundamental degrees of freedom contributing to the response dynamics freeze for all time and for arbitrary initial state. Also, since the condition of freezing involves only the drive parameters and not the quantization of the momentum (i.e., the system size), our simulation with a small (3-spin) chain captures all salient features of the freezing phenomenon predicted for the infinite chain. Using optimal control techniques, we realize high-fidelity cosinemodulated drive, and observe nonmonotonic freezing of magnetization at specific frequencies of modulation. Time evolution of the excitations in momentum space has been tracked directly through magnetization measurements.
\end{abstract}

DOI: 10.1103/PhysRevB.90.174407

\section{INTRODUCTION}

The study of nonequilibrium dynamics has emerged as one of the central topics in quantum many-body physics, thanks to the recent advancements in various experimental techniques [1-10]. In particular, the paradigm of periodically driven many-body systems has hosted quite a few interesting theoretical (see, e.g., [11-23]) and experimental results (see, e.g., [24-29]) in recent days. We add to this list, experimental verification of a nonequilibrium freezing phenomenon, where quantum interference brings about a surprising counterclassical scenario [30].

In general, response of a simple driven system may "freeze" if the drive rate is much faster compared to the characteristic relaxation rate of the system. The mechanism is intuitively simple - the system does not get sufficient time to change itself significantly within the duration of the drive. Thus, the faster the drive is, the more frozen is the response. This intuition is manifested in many celebrated results in both classical and quantum physics, such as the Kibble-Zurek scaling law for defect generation in classical [31,32] and quantum [33-35] phase transitions, Landau-Zener excitation probability [36,37], and the phenomenon of classical dynamical hysteresis [38], to name a few. It forms the basis of the so called sudden approximation in quantum mechanics [39] and its more elaborate descendent, the adiabatic-impulse approximation which applies to classical and quantum systems alike [32,40]. For a periodically driven system, this intuition implies a stronger freezing for a higher frequency of the drive.

However, in certain cases this intuition may grossly fail due to quantum interference of excitation amplitudes. In our experiment we demonstrate one such phenomenon, dubbed dynamical many-body freezing [20,30,41-44], where repeated quantum interference results in strongly nonmonotonic freezing behavior with respect to the driving rate in a

\footnotetext{
*These authors contributed equally to this work.

${ }^{\dagger}$ mahesh.ts@iiserpune.ac.in

†arnab.das.physics@gmail.com
}

periodically driven quantum magnet, contrasting the above intuitive scenario. Most surprisingly, absolute freezing (i.e., freezing of all excitations contributing to the response dynamics) is observed for certain particular values of drive parameters in a large class of integrable models for various kinds of periodic drive [30,41-44].

This phenomenon is akin to the well-known single-particle phenomena of dynamical localization [45] and coherent destruction of tunneling [46], where a quantum particle is kept localized in space under an externally applied periodic drive. But it is not only a many-body extension of dynamical localization; it is also a much more drastic version of it: here the freezing is not limited to initial states localized in real space as happens in dynamical localization, but applies for any initial state in the Hilbert space [30].

Major experimental work has been done in recent years (see, e.g., [47,48]) in probing equilibrium quantum critical properties, e.g., locating a quantum critical point of an Ising magnet using nuclear magnetic resonance (NMR). Here we take a major step forward by experimentally demonstrating a nonequilibrium phenomena in a coherently driven many-body system, where quantum coherence plays a surprising role as we discuss here.

The rest of the paper is organized as follows. First, we outline the theory and the phenomenology of the dynamical many-body freezing, particularly for our finite-size experimental system. Then we discuss the experimental setup and realization of the phenomenon followed by the result and its analysis, and finally we conclude with an outlook.

\section{DYNAMICAL MANY-BODY FREEZING IN ISING CHAIN: THEORY}

\section{A. Review of the phenomenon in an infinite chain}

First we review the phenomenon in an infinite onedimensional Ising ring that is being subjected to a transverse periodic-driving field of amplitude $h_{0}$, frequency $\omega$ (period $\tau=2 \pi / \omega)$, and represented by the Hamiltonian $\mathcal{H}(t)=$ $-\frac{1}{2}\left[\mathcal{J} \sum_{i=1}^{\infty} \sigma_{i}^{z} \sigma_{i+1}^{z}+h_{0} \cos (\omega t) \sum_{i=1}^{\infty} \sigma_{i}^{x}\right]$. Here $\mathcal{J}$ is the 
strength of the nearest-neighbor Ising interaction and $\sigma_{i}^{x / z}$ are Pauli matrices. A dynamical freezing parameter $Q$, defined as the long-time average of magnetization in the $x$ direction, $m^{x}(t)$, is used for quantifying the degree of freezing via the anomalous dc response:

$$
Q=\lim _{\mathcal{T} \rightarrow \infty} \frac{1}{\mathcal{T}} \int_{0}^{\mathcal{T}} m^{x}(t) d t,
$$

where $\mathcal{T}$ is the duration of total evolution.

Suppose that at $t=0$ the system is at a state completely polarized in the $x$ direction. Then the adiabatic limit $(\omega / L \rightarrow$ 0 , where $L$ is the system size) corresponds to $Q=0$ in the $L \rightarrow \infty$ limit (since the average of the transverse field is zero over each cycle), and the impulse or "freezing" limit of $(\omega \rightarrow \infty)$ corresponds to $Q=1 / 2$. Classical intuition of "faster drive means lesser time to react" would suggest a monotonic behavior of $Q$ as a function of $\omega$ interpolating between these two regimes. But quantum interference leads to a strongly nonmonotonic freezing behavior for $\omega \gg \mathcal{J}$. In the limit $L \rightarrow \infty, Q$ is accurately given by a closed-form analytical formula [30]

$$
Q=\frac{1}{1+\left|J_{0}\left(2 h_{0} / \omega\right)\right|},
$$

where $J_{0}(\cdot)$ denotes the ordinary Bessel function of order 0 .

The gist of the result in Eq. (2) is summarized below (see Fig. 1). First, the average magnetization $Q$, which quantifies the degree of freezing, is a highly nonmonotonic function of $\omega$ in contrast to what is expected intuitively. Second, there are certain values of the ratio of the amplitude $h_{0}$ and frequency $\omega$ of the driving at which

$$
J_{0}\left(2 h_{0} / \omega\right)=0
$$

we have $Q=1$, which means the time average of $m^{x}(t)$ is equal to the initial $m^{x}$, which was set to the maximum value

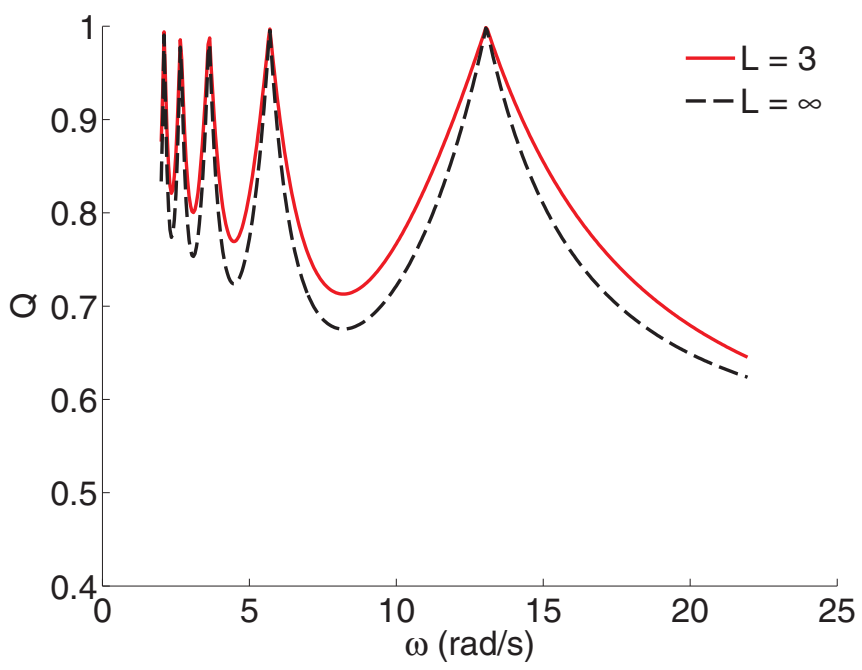

FIG. 1. (Color online) Comparison of analytical results for $Q$ obtained for the $\omega \gg \mathcal{J}$ limit for $L \rightarrow \infty$ [Eq. (2)] and $L=3$ [Eq. (12)] for $h_{0}=5 \pi \mathrm{rad} / \mathrm{s}, \mathcal{J}=h_{0} / 20$. Strong nonmonotonic freezing behavior with respect to the drive frequency $\omega$ is visible, in contrast to the intuition of faster drive $\Rightarrow$ lesser time to respond $\Rightarrow$ more frozen response. Almost absolute freezing $Q \approx 1$ is visible for certain special values of $\omega, h_{0}$ satisfying the freezing condition [Eq. (3)]. Our experiment captures all these features remarkably well (Fig. 4). (unity) which $m^{x}$ can assume. This is possible if $m^{x}$ has remained absolutely frozen for all time. Analysis shows this happens because population dynamics of each fundamental degree of freedom (which happens to be independent fermionic excitations in momentum space) freezes absolutely [30].

\section{B. Dynamical many-body freezing for $L=3$}

In this subsection we discuss the fate of dynamical freezing for a chain of size $L=3$, which is the size of our experimentally realized sample. Analysis of this particular case is important not only for matching with the experiment, but it has an important finite-size effect which vanishes in the $L \rightarrow \infty$ limit. Nevertheless, we show that $L=3$ results in capturing all the essential features of the infinite-size limit. Moreover, this analysis allows us to illustrate the pivotal role quantum interference plays in the phenomenon.

The Hamiltonian we consider is

$$
\mathcal{H}=-\frac{1}{2}\left[\mathcal{J} \sum_{i}^{3} \sigma_{i}^{z} \sigma_{i+1}^{z}+h_{0} \cos (\omega t) \sum_{i}^{3} \sigma_{i}^{x}\right],
$$

with periodic boundary condition $\sigma_{4}=\sigma_{1}$. The eigen-problem of the above Hamiltonian can be solved analytically by Jordan-Wigner transformation followed by Fourier transform for any $L$ (see, e.g., $[44,49,50]$ ). The above Hamiltonian can be mapped to independent fermionic Hamiltonians in momentum space such that only fermions with equal but opposite momenta $\pm k$ are coupled: $\mathcal{H}(t)=\prod_{k>0} H_{k}$, with

$$
H_{k}=-E_{k}\left(c_{k}^{\dagger} c_{k}-c_{-k} c_{-k}^{\dagger}\right)+i \Delta_{k}\left(c_{-k} c_{k}-c_{k}^{\dagger} c_{-k}^{\dagger}\right) \text {, }
$$

where $E_{k}=h_{0} \cos \omega t+\mathcal{J} \cos k, \Delta_{k}=\mathcal{J} \sin k$, and $h_{x}(t)=$ $h_{0} \cos \omega t$. The quantization of $k$ depends on the parity of the fermion number (i.e., whether it is odd/even) which is conserved throughout the dynamics (although the parity in the ground state depends on the sign of $h_{x}$, which means the ground state can never be followed adiabatically; see [51]. From a dynamical point of view, this happens because one of the three modes remains absolutely frozen irrespective of how slow the drive is, as discussed below.) Here for definiteness, we start with the ground state of $\mathcal{H}$ for any parameter value, which will always have an even number of fermions. This leads to the quantization $k=-\pi, \pm \pi / 3$ (given our choice of our Brillouin zone). Now we note that $k=-\pi$ has no partner to pair with, while the only pair formed is for $k= \pm \pi / 3$. Thus we can work with a Hilbert space spanned by the vectors $\left\{\left[\left|0_{-\pi / 3}, 0_{\pi / 3}\right\rangle,\left|1_{-\pi / 3}, 1_{\pi / 3}\right\rangle\right] \otimes\left[\left|0_{-\pi}\right\rangle,\left|1_{-\pi}\right\rangle\right]\right\}$. In this representation the initial state fully polarized in the $+x$ direction reads $|\psi(0)\rangle=\left|1_{-\pi / 3}, 1_{\pi / 3}\right\rangle \otimes\left|1_{-\pi}\right\rangle$. Henceforth we denote $\left|0_{-\pi / 3}, 0_{\pi / 3}\right\rangle$ by $\left|0_{\pi / 3}\right\rangle$ and $\left|1_{-\pi / 3}, 1_{\pi / 3}\right\rangle$ by $\left|1_{\pi / 3}\right\rangle$. Noting that the $k=-\pi$ mode has no dynamics, the state at any time can be given by

$$
|\psi(t)\rangle=\left[u_{\pi / 3}\left|0_{\pi / 3}\right\rangle+v_{\pi / 3}\left|1_{\pi / 3}\right\rangle\right] \otimes\left|1_{-\pi}\right\rangle .
$$

The time-dependent transverse magnetization in this notation reads (for the initial state fully polarized in the $+x$ direction)

$$
m^{x}(t)=\frac{2}{3} \sum_{k=-\pi, \pm \pi / 3}\left\langle c_{k}^{\dagger} c_{k}\right\rangle-1=\frac{4}{3}\left|v_{\pi / 3}(t)\right|^{2}-\frac{1}{3} .
$$


Now $\left\{u_{\pi / 3}, v_{\pi / 3}\right\}$ (with a suitably adjusted common phase factor; see [56]) satisfies the following time-dependent Schrödinger equation:

$$
i \frac{\partial}{\partial t}\left[\begin{array}{c}
u_{\pi / 3}(t) \\
v_{\pi / 3}(t)
\end{array}\right]=\left[\begin{array}{cc}
E_{\pi / 3} & i \Delta_{\pi / 3} \\
-i \Delta_{\pi / 3} & -E_{\pi / 3}
\end{array}\right]\left[\begin{array}{c}
u_{\pi / 3}(t) \\
v_{\pi / 3}(t)
\end{array}\right],
$$

where $\quad E_{\pi / 3}=h_{0} \cos (\omega t)+\mathcal{J} \cos (\pi / 3)$ and $\Delta_{\pi / 3}=$ $\mathcal{J} \sin (\pi / 3)$. The exact solution of the above $2 \times 2$ matrix equation is not known but the analytical solution can be obtained under a rotating wave approximation in the fast-driving regime $(\omega \gg 2 \mathcal{J})$ [30]. For the initial condition mentioned above, the solution for $\left|v_{\pi / 3}\right|^{2}$ reads

$$
\begin{aligned}
\left|v_{\pi / 3}\right|^{2} & =1-A_{\pi / 3}^{2} \sin ^{2}\left(\phi_{\pi / 3} t\right), \text { where } \\
\phi_{\pi / 3} & =|\mathcal{J}| \sqrt{J_{0}^{2}\left(2 h_{0} / \omega\right) \sin ^{2}\left(\frac{\pi}{3}\right)+\cos ^{2}\left(\frac{\pi}{3}\right)}, \\
A_{\pi / 3} & =\frac{J_{0}\left(2 h_{0} / \omega\right) \mathcal{J} \sin \left(\frac{\pi}{3}\right)}{\phi_{\pi / 3}} .
\end{aligned}
$$

This gives

$$
\begin{array}{r}
m^{x}(t)=Q(L=3)+\frac{2 A_{\pi / 3}^{2}}{3} \cos \left(2 \phi_{\pi / 3} t\right), \\
Q(L=3)=1-\frac{2}{3} A_{\pi / 3}^{2}=\frac{1+\left|J_{0}\left(2 h_{0} / \omega\right)\right|}{1+3\left|J_{0}\left(2 h_{0} / \omega\right)\right|} .
\end{array}
$$

Comparing this with Eq. (2) we see that $Q$ has maximum value (unity) in both cases of $L=3$ and $L \rightarrow \infty$ for $J_{0}\left(2 h_{0} / \omega\right)=0$. Also, since $\left|J_{0}\left(2 h_{0} / \omega\right)\right| \leqslant 1, Q(L=3) \geqslant Q(L \rightarrow \infty)$. Our experimental results accurately capture these features (Fig. 4).

\section{EXPERIMENTAL REALIZATION}

We simulate the above freezing phenomenon in the 3 -spin chain given by Eq. (4). Going beyond the theoretically explored cases of pure initial states, we also demonstrate strong freezing for a mixed initial state. We use the three spin-1/2 ${ }^{19} \mathrm{~F}$ nuclei of trifluoroiodoethylene dissolved in acetone-D6 as our 3-spin Ising system. The molecular structure of the system is shown in Fig. 2(a). All the experiments described below were carried out using a Bruker $500 \mathrm{MHz}$ NMR spectrometer at an ambient temperature of $290 \mathrm{~K}$. The simulation involves preparing a certain initial state, evolving it under the Hamiltonian $\mathcal{H}(t)$ for a total time $T$, while measuring the response of the system in intervals of time $\tau$.

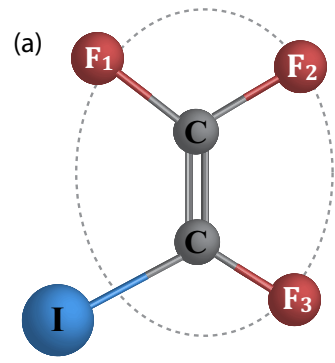

(b)

\begin{tabular}{|c|c|c|c|}
\hline $\mathbf{F}_{1}$ & $\mathbf{F}_{\mathbf{2}}$ & $\mathbf{F}_{3}$ & \\
\hline 11860.8 & 69.9 & 47.4 & $\mathbf{F}_{1}$ \\
\hline & 0 & -128.3 & $\mathbf{F}_{2}$ \\
\hline & & -17379.1 & $\mathbf{F}_{3}$ \\
\hline
\end{tabular}

FIG. 2. (Color online) (a) Molecular structure and (b) chemical shifts (diagonal terms) and $J$ coupling (off-diagonal terms) in $\mathrm{Hz}$ of trifluoroiodoethylene.
The thermal equilibrium state for the NMR spin system is $\left(I / 8+\epsilon \sum_{i=1}^{3} \sigma_{i}^{z} / 2\right)$ where $I$ represents uniform background population, which does not evolve under unitary transformations and is henceforth ignored. Here $\epsilon$ is the polarization of the spin (see [52]). The response of the system measured as transverse magnetization is given by

$$
m^{x}(t)=\frac{1}{m_{0}} \operatorname{Tr}\left[\rho(t)\left(\sum_{i=1}^{3} \sigma_{i}^{x} / 2\right)\right] .
$$

Here $\rho(t)$ is the instantaneous density matrix of the driven system and $m_{0}$ is a normalization factor [52] given by the maximum possible transverse magnetization; i.e., $m_{0}=$ $\operatorname{Tr}\left[\left(\sum_{i=1}^{3} \sigma_{i}^{x} / 2\right)\left(\sum_{i=1}^{3} \sigma_{i}^{x} / 2\right)\right]$. We performed a set of experiments for each of the following two different initial states. The first initial state is $\rho(0)=\sum_{i=1}^{3} \sigma_{i}^{x} / 2$ [i.e., $m^{x}(0)=1$, fully polarized in the $+x$ direction] obtained by applying a global $R_{y}(\pi / 2)=\exp \left\{-i(\pi / 2) \sum_{i}^{3} \sigma_{i}^{y} / 2\right\}$ rotation on the thermal state. This state is approximately the ground state of our Hamiltonian (4) at $t=0$, if $h_{0} \gg \mathcal{J}$. The second initial state is $\rho(0)=\sum_{i=1}^{3}\left(\sigma_{i}^{x} / 2+\sigma_{i}^{z} \sqrt{3} / 2\right) / 2$ [i.e., $m^{x}(0)=0.5$ ] obtained by applying a global $R_{y}(\pi / 6)$ rotation on the thermal state. Note that this state is not the ground state of Hamiltonian (4). For convenience, we refer to these two sets of experiments by their initial magnetization values, $m^{x}(0)$.

The internal Hamiltonian for the NMR system is given by

$$
\mathcal{H}_{\text {int }}=-\pi \sum_{i=1}^{3} v_{i} \sigma_{i}^{z}+\frac{\pi}{2} \sum_{\substack{i, j=1 \\ i<j}}^{3} J_{i j} \sigma_{i}^{z} \sigma_{j}^{z},
$$

where the first term represents the Zeeman part and the second term represents the spin-spin interaction part. Here $v_{i}$ is the resonance frequency of the $i$ th spin in the rotating frame, and $J_{i j}$ is the strength of the indirect spin-spin interaction between spins $i$ and $j$. The parameters of the internal Hamiltonian for the above spin system are shown in Fig. 2(b).

In order to simulate the time-dependent Hamiltonian $\mathcal{H}(t)$ using $\mathcal{H}_{\text {int }}$, we need to (i) cancel the evolution under Zeeman interaction, (ii) bring out an effective Ising interaction of strength $\mathcal{J}$, and (iii) add the oscillatory drive $\left(-h_{0} / 2\right) \cos (\omega t)$ along the $x$ direction. Equivalently, the evolution under the Hamiltonian $\mathcal{H}(t)$ in Eq. (4) is realized by designing an RF modulation using a standard optimal control technique such that the effective evolution for a small time step $\delta t$ is as close to $\exp (-i \mathcal{H} \delta t)$ as possible [53]. The procedure involves discretizing time duration $T$ into $M$ equal steps each of duration $\delta t=T / M$ such that the propagators for the discretized Hamiltonian are

$$
U_{k}=\exp [-i \delta t \mathcal{H}(k \delta t)],
$$

for $k \in\{1,2, \ldots, M\}$. The overall evolution for a time $k \delta t$ is given by the unitary $U(k \delta t)=U_{k} U_{k-1} \cdots U_{2} U_{1}$. The response $m^{x}(t)$ [refer to Eq. (13)] is measured at time instants $t=n \tau$, for $n=0,1,2, \ldots, N$, where $N=T / \tau$ corresponds to the last measurement. Therefore we only need to generate the propagators $U(n \tau)$. We used the gradient ascent pulse engineering (GRAPE) method to design amplitude- and phase-modulated radio frequency $(\mathrm{RF})$ pulses which effectively realize these propagators [53] 

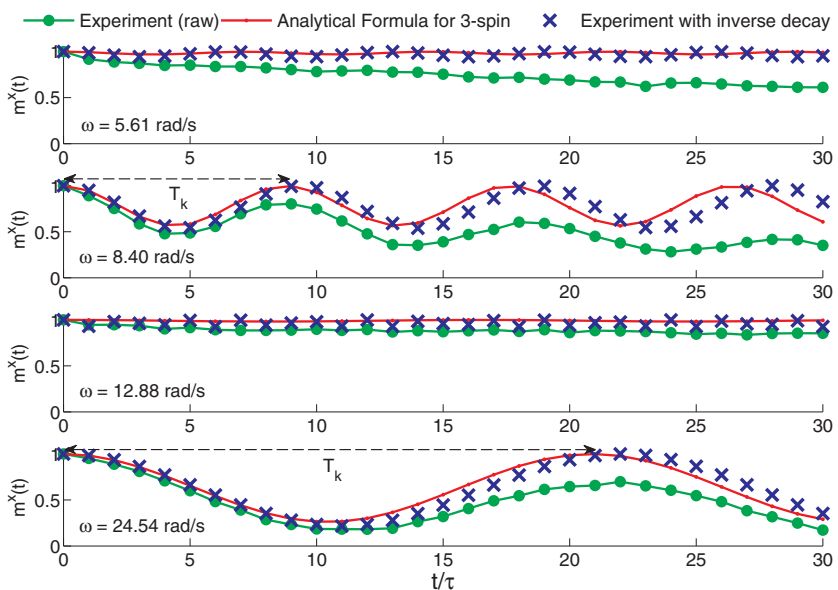

FIG. 3. (Color online) Evolution of $m^{x}$ with time for $m^{x}(0)=1$. The period $(\tau)$ of oscillation of $m^{x}$ visible from the experimental data (marked for $\omega=8.40,24.54 \mathrm{rad} / \mathrm{s}$ ) corresponds to the frequency $2 \phi_{k}$ (period $T_{k}=2 \pi / \phi_{k}$ ) of population oscillation for the fundamental excitation in momentum space for $k=\pi / 3$ given in Eq. (9). The analytical curves represent Eq. (11).

The dynamical freezing parameter for this discretized set of measurements is defined as $Q=\frac{1}{N+1} \sum_{n=0}^{N} m^{x}(n \tau)$. The experiments were carried out for 24 different values of $\omega$ ranging from 3.59 to $24.54 \mathrm{rad} / \mathrm{s}$. In each case we chose the measurement interval as $\tau=2 \pi / \omega$, the period of the driving frequency. We discretized each cycle of the periodic Hamiltonian $\mathcal{H}(t)$ into 11 steps and constructed the propagators $U(n \tau)$ for $N=30$ cycles; i.e., $n=0,1,2, \ldots, N$. Here the maximum number of cycles was limited by the duty cycle of the RF channel. The one-cycle propagator $U(\tau)=$ $U_{11} U_{10} \cdots U_{2} U_{1}$, where $U_{k}$ s are given by Eq. (15). Since $\mathcal{H}(t)$ has a period of $\tau, U(n \tau)=U^{n}(\tau)$. All numerically generated GRAPE pulses were optimized against RF inhomogeneity and had an average Hilbert-Schmidt fidelity greater than or equal to 0.99 . For the 30th cycle, the overall duration of the GRAPE pulses were ranging between $48 \mathrm{~ms}$ to $300 \mathrm{~ms}$ for different $\omega$ values.

The overall experimental sequence can be represented by the unitary $U(n \tau) \cdot R_{y}(\pi / 2)$ for $m^{x}(0)=1$ and $U(n \tau)$. $R_{y}(\pi / 6)$ for $m^{x}(0)=0.5$. The net-transverse magnetization corresponding to the observable $\sum_{i=1}^{3} \sigma_{i}^{x} / 2$ was measured as the intensity of the real part of the NMR signal acquired in a quadrature mode and is normalized by the reference signal obtained with only $R_{y}(\pi / 2)$ pulse [52].

Results. The main result of our experiment is summarized in Figs. 3-5. In Fig. 3 the dynamics of $m^{x}$ as a function of time is shown for different values of $\omega$, and for parameters $h_{0}=5 \pi$ and $\mathcal{J}=h_{0} / 20$ (in rad/s). The freezing of the dynamics is clearly visible close to the theoretically predicted values of $\omega$ $\left[\approx 5.61\right.$ and $12.88 \mathrm{rad} / \mathrm{s}$, corresponding to $\left.J_{0}\left(2 h_{0} / \omega\right) \approx 0\right]$. The oscillation of $m^{x}(t)$ with time allows us to directly read off the frequency of the population oscillation of the underlying fermionic mode $k=\pi / 3$ in the momentum space using Eq. (11). However, from the raw experimental data (green line, filled circles), we see that $m^{x}$ decays steadily even under the maximal freezing conditions. This decay is due to decoherence, transverse relaxation $\left(T_{1 \rho}\right)$ [54], as

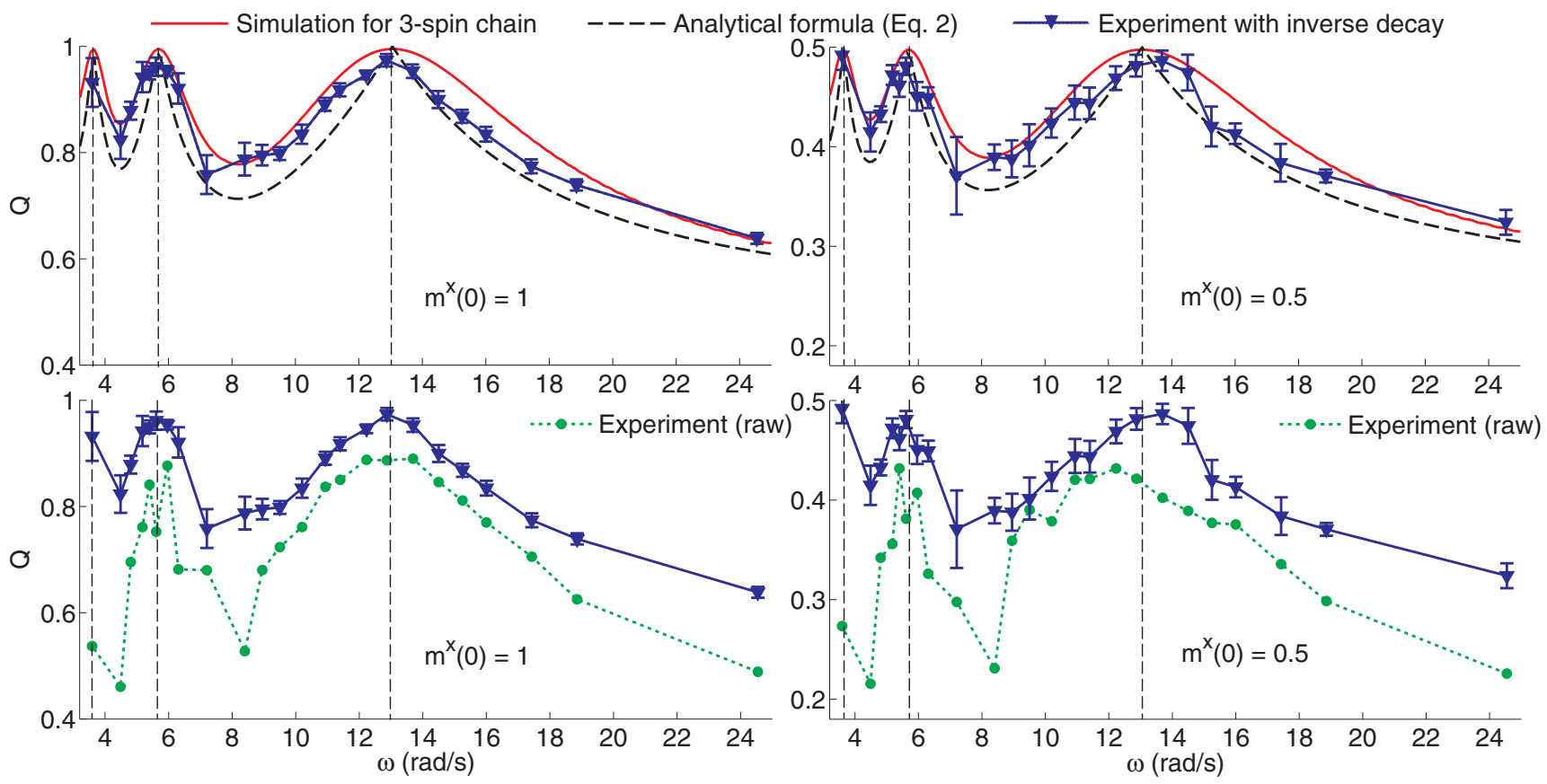

FIG. 4. (Color online) $Q$ vs $\omega$ for different initial conditions: Left panels for the initial state corresponding to $m^{x}(0)=1$, right panels for the initial state corresponding to $m^{x}(0)=0.5$. Top frames compare the final experimental data (with inverse decay correction) for 3 -spins with the theoretical results for $L=3$ and $L \rightarrow \infty$. Simulation for $L=3$ is done by solving the exact time-dependent Schrödinger equation numerically. $Q$ is obtained by measuring $m^{x}$ after each cycle and carrying out the stroboscopic average over 30 cycles (as done in the experiment). The vertical lines indicate the position of the freezing peaks. The bottom frames compare raw experimental data with the decay-corrected data. Both the data capture all the essential features of those predicted theoretically for $L \rightarrow \infty$. 


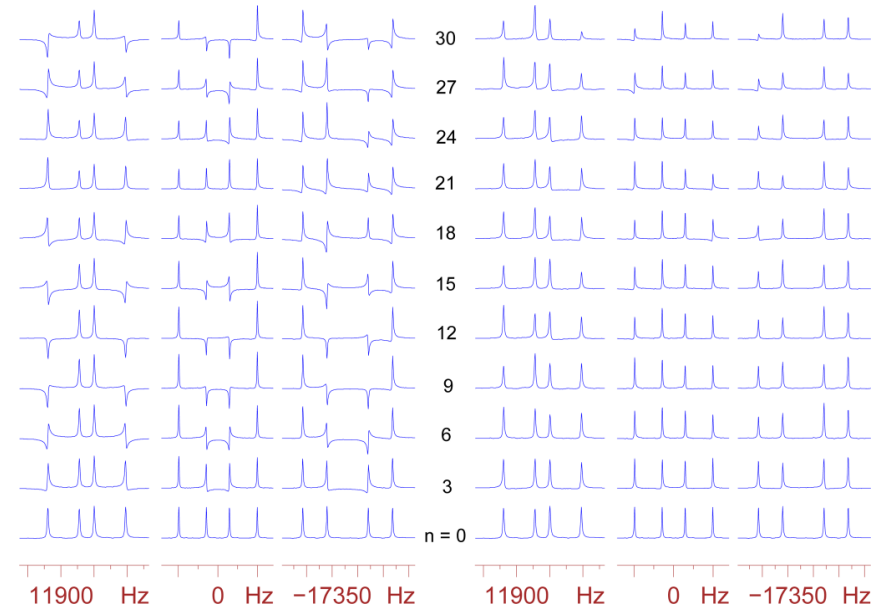

FIG. 5. (Color online) ${ }^{19} \mathrm{~F}$ spectra corresponding to a nonfreezing condition (left column; $\omega=24.54 \mathrm{rad} / \mathrm{s}$ ) and a freezing condition (right column; $\omega=5.61 \mathrm{rad} / \mathrm{s}$ ) for $m^{x}(0)=1$. Only spectra for the indicated time instants $(n=t / \tau)$ are shown. In contrast to the classical intuition, the response is clearly seen to be much strongly frozen for the much lower driving frequency $(\omega=5.61 \mathrm{rad} / \mathrm{s})$ due to quantum interference, as predicted by the theory.

well as decay caused by the spatial inhomogeneities in RF amplitudes [29]. For all other values of $\omega$, within our chosen $\omega$ range, we see oscillations in $x$ magnetization in addition to the decay. In order to confirm this, we took into account the effect of decay by fitting the experimental data points $m^{x}(t)$ with the standard decay functions; i.e., $m^{x}(t)=\alpha+[\beta+\gamma \cos (c t)] e^{-t / T_{d}}$, where $\alpha, \beta, \gamma, c$, and $T_{d}$ are the fitting parameters. Here $T_{d}$ is the decay constant and is obtained by the fitting $m^{x}(t)$ to the above function [52]. The resulting data points are shown in Fig. 3 as crosses.

Figure 4 shows $Q$ vs $\omega$ plots again for experimental parameters $h_{0}=5 \pi$ and $\mathcal{J}=h_{0} / 20$ (in rad/s). The peaks represent the freezing points. For numerical simulation of the 3 -spin Ising chain, we observe three freezing points in this range, viz. at $\omega=3.59,5.61$, and $12.88 \mathrm{rad} / \mathrm{s}$. These points are very close to the one predicted by the analytical formula [Eq. (3)], which gives freezing values to be $\omega=3.63,5.69$, and $13.06 \mathrm{rad} / \mathrm{s}$ in our case. Comparison of the experimental data after inverse-decay correction with the exact numerical simulation for the $Q$ lines exhibits striking agreement. The raw experimental data exhibit an overall downward shift of the experimental line due to the $T_{d}$ decay, though the basic features (particularly, the peak positions) remain same. We also compare the finite-size result with the infinite-size analytical formula (2) in Fig. 4. Interestingly, not only the freezing peaks, but also the trend of most of the $Q$ vs $\omega$ profile for our 3-spin system driven over 30 cycles matches fairly well with that of an infinite chain driven over infinite time.

In Fig. 5, we show the experimentally obtained spectra for $m^{x}(0)=1$ at time instants $0,3 \tau, 6 \tau, \ldots, 30 \tau$, for two driving frequencies (i) $\omega=24.54 \mathrm{rad} / \mathrm{s}$ not satisfying the freezing condition (left column) and (ii) $\omega=5.61 \mathrm{rad} / \mathrm{s}$ satisfying the freezing condition (right column). The spectra at time instant $n=0$ correspond to the state completely polarized in the $+x$ direction. The phase oscillations of the spectral lines in the nonfreezing case (left column) can be clearly noticed, while the freezing spectra (right column) remain in-phase.

\section{A. Role of quantum interference: Amplitude vs probability}

It is interesting to consider the crucial role of quantum interference in this phenomenon. The nonmonotonic nature of the freezing phenomena, including the appearance of maximal freezing peaks, is a consequence of quantum interference, as indicated in [30]. Here we explicitly demonstrate the role of quantum interference behind the key features of the freezing behavior. To this end we analyze the problem using another approach based on repeated calculation of Landau-Zener-like excitation, which has been employed successfully to estimate certain aspects of a repeated quench dynamics in a similar model in the presence of decoherence after each sweep [55]. The role of quantum interference in repeated quenches has been studied earlier [22]. We demonstrate how such an approach (based on counting of probabilities rather than amplitudes) fails to explain even the qualitative features of the freezing phenomenon. Suppose we start from the initial state $\left\{u_{\pi / 3}(0) \approx 0, v_{\pi / 3}(0) \approx 1\right\}$, and make $2 m$ number of half cycles and find that we are in a state $\left\{u_{\pi / 3}(2 m), v_{\pi / 3}(2 m)\right\}$, where $P_{k}(2 m)=\left|v_{\pi / 3}(2 m)\right|^{2}$ (see Fig. 6). Now we want to estimate the probability $\left|v_{\pi / 3}(2 m+1)\right|^{3}$ of being in the state $\left|1_{\pi / 3}\right\rangle$ after the subsequent [i.e., $(2 m+1)$ th] half sweep is made. We can come up with an estimate based on calculating transition probabilities if we are supplied with the excitation probability $P_{e x}$, which is the probability that we start with the states $\left|1_{\pi / 3}\right\rangle$ before starting a half cycle and end up with the same state $\left|1_{\pi / 3}\right\rangle$ after completing the half cycle. The name excitation probability is motivated by the fact that since $h_{0} \gg$ $\mathcal{J}$, for $h^{x}=+h_{0}$ the ground state (to a good approximation) corresponds to $\left|1_{\pi / 3}\right\rangle$ (left end of Fig. 6), while for $h^{x}=-h_{0}$ the ground state approximately corresponds to $\left|0_{\pi / 3}\right\rangle$ (right end of the Fig. 6). Due to the symmetry of the problem, this is also

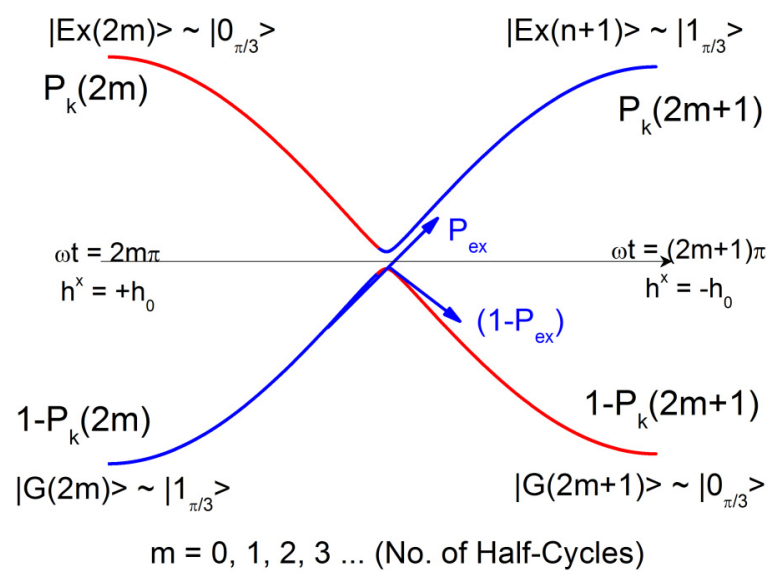

FIG. 6. (Color online) Plot of energies of the instantaneous ground state (denoted by $|G\rangle$ ) and excited state $(|E x\rangle)$ of the $2 \times 2$ Hamiltonian [Eq. (8)] as a function of $\omega t$. Our first sweep $(m=0)$ starts from the left end with the initial state $|\psi(0)\rangle_{\pi / 3}=\left\{u_{\pi / 3}(0)=\right.$ $\left.0 ; v_{\pi / 3}(0)=1\right\} \approx|G(0)\rangle$. $P_{e x}$ denotes the probability that starting from this initial state the system ends up with the same state (i.e., $\left.\left\{u_{\pi / 3}=0 ; v_{\pi / 3}=1\right\}\right)$ at the end of half a cycle, instead of following the drive adiabatically and ending up with the ground state $\left(\approx\left|0_{\pi / 3}\right\rangle\right)$ of the final Hamiltonian. 


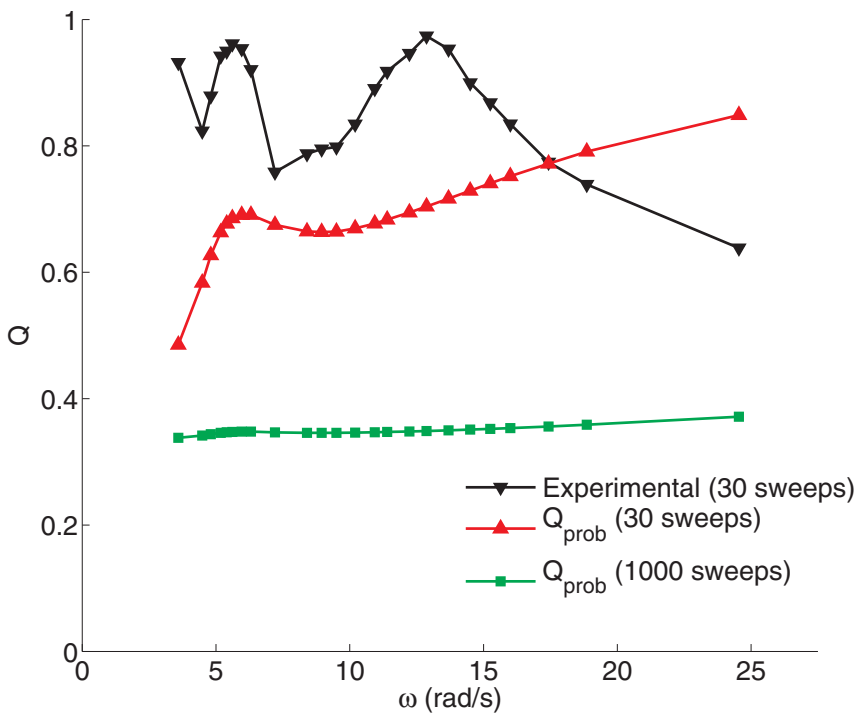

FIG. 7. (Color online) Qualitative feature of the theory that uses only transition probabilities between the states $\left|u_{\pi / 3}\right\rangle,\left|v_{\pi / 3}\right\rangle$ after each half cycle, neglecting the phase between them. This illustrates the importance of quantum interference-the role those phases play in shaping the qualitative characteristics of the freezing phenomenon. The full quantum treatment provides excellent agreement with the experimental data (Fig. 4). The results correspond to experimental parameter values $h_{0}=5 \pi, \mathcal{J}=h_{0} / 20(\mathrm{in} \mathrm{rad} / \mathrm{s})$.

the probability that we end up in the ground state starting from the excited state. Since $h_{0} \gg \mathcal{J} \cos k$, we can approximately use the same $P_{e x}$ for the reverse sweep (using a different one for the reverse sweep does not change the conclusion in any qualitative way). Now taking the excitation probabilities into account we find the following recursion relation:

$$
\left|v_{\pi / 3}(2 m+1)\right|^{2}=\left|v_{\pi / 3}(2 m)\right|^{2}\left(2 P_{e x}-1\right)+1-P_{e x},
$$

where $m=0$ denotes the initial state (starting from the left). Solving above relation one gets

$$
\left|v_{\pi / 3}(2 m+1)\right|^{2}=\frac{1}{2}+\left(2 P_{e x}-1\right)^{2 m}\left[\left|v_{\pi / 3}(0)\right|^{2}-\frac{1}{2}\right] .
$$

Thus $\left|v_{\pi / 3}(2 m+1)\right|^{2} \rightarrow \frac{1}{2}$ as $m \rightarrow \infty$. This implies $Q \rightarrow 0$ regardless of $\omega$ as $m \rightarrow \infty$ as $L \rightarrow \infty$. In our case $(L=3)$ this would give [using Eq. (11)]

$$
\lim _{N_{s} \rightarrow \infty} Q_{\operatorname{Prob}}\left(N_{s}\right)=\lim _{N_{s} \rightarrow \infty} \frac{1}{N_{s}} \sum_{n=0}^{N_{s}} m^{x}(t=n \tau)=\frac{1}{3},
$$

where $Q_{\text {Prob }}$ denotes $Q$ calculated using transition probabilities (instead of amplitudes) as described above. This shows that the variation of $Q$ with $\omega$ and $h_{0}$ is itself a result of repeated quantum interference between the phases gathered after the half cycles.
We see a qualitative difference between the experimental results (which agrees with the correct quantum treatment, as shown in Fig. 4) and the above probability-based calculation. In Fig. 7 we show an explicit comparison of the experimental result with the numerical result calculated using Eqs. (17) and (11) for the experimental parameter values. $Q_{\text {Prob }}$ is also calculated stroboscopically over 30 cycles as has been done in the experiment. $P_{e x}$ is calculated exactly by evolving the system numerically for half a cycle for each value of $\omega$. $m^{x}(n)$ is measured after each complete cycle and averaged over cycles to calculate $Q_{\text {Prob }}$ (as is done in the experiment). The result shows a monotonic behavior for $Q_{\text {Prob }}$ for 30 cycles (orange line, triangles) as a function of $\omega$ for large $\omega$, only with a reminiscence of the nonmonotonic behavior close to the first peak. However, for $Q_{\text {Prob }}$ averaged over a larger number of sweeps this profile tends to flatten out completely, as demonstrated by the plot of $Q_{\text {Prob }}$ for 1000 sweeps (green line, squares). In that case $Q \approx 3$ independently of $\omega$ as predicted by Eq. (18). The probability-based calculations that neglect the phase between $\left|u_{\pi / 3}\right\rangle$ and $\left|v_{\pi / 3}\right\rangle$ thus produce a qualitatively wrong result, even within our experimental window of time and small system size. This illustrates the significance of quantum interference in the freezing phenomenon.

\section{B. Conclusion and outlook}

In our NMR experiments we have demonstrated that repeated quantum interference can strongly freeze the magnetization dynamics of a periodically driven Ising chain for certain particular values of the driving amplitude and the frequency, and confirmed the phenomenon of dynamical many-body freezing with excellent quantitative accuracy for both initial states. This is a major step forward, where a surprising nonequilibrium manifestation of quantum interference has been accurately demonstrated experimentally. With further experimental accuracies, complete freezing of certain quantities against unwanted evolution in a system of interacting qubits (Ising spins) might be possible by imposing strong Ising interactions between the qubits and a suitable periodic drive. Such a coherent control mechanism might inspire useful technologies as has been done in the past. For example, the average Hamiltonian theory developed by Waugh and co-workers founded high-resolution solid-state NMR [28]. It involves coherent driving of spin systems via a time-dependent Hamiltonian, leading to an effective evolution under desired interactions. A constant drive with a strong transverse field, called spin lock, is used in many of the NMR experiments to create an effective transverse Hamiltonian in the rotating frame [29].

\section{ACKNOWLEDGMENTS}

The authors are grateful to B. K. Chakrabarti, A. Kumar, A. Lazarides, R. Moessner, and S. S. Roy for useful discussions. This work was partly supported by DST (India), SR/S2/LOP-0017/2009.
[1] C. H. Tseng, S. Somaroo, Y. Sharf, E. Knill, R. Laflamme, T. F. Havel, and D. G. Cory, Phys. Rev. A 62, 032309 (2000).
[2] L.-A. Wu, M. S. Byrd, and D. A. Lidar, Phys. Rev. Lett. 89, 057904 (2002). 
[3] Y. S. Weinstein, S. Lloyd, J. Emerson, and D. G. Cory, Phys. Rev. Lett. 89, 157902 (2002).

[4] C. Negrevergne, R. Somma, G. Ortiz, E. Knill, and R. Laflamme, Phys. Rev. A 71, 032344 (2005).

[5] K. R. Brown, R. J. Clark, and I. L. Chuang, Phys. Rev. Lett. 97, 050504 (2006).

[6] X. Yang, A. M. Wang, F. Xu, and J. Du, Chem. Phys. Lett. 422, 20 (2006).

[7] G. Roumpos, C. P. Master, and Y. Yamamoto, Phys. Rev. B 75, 094415 (2007).

[8] I. Bloch, J. Dalibard, and S. Nascimbène, Nat. Phys. 8, 267 (2012).

[9] I. Bloch, J. Dalibard, and W. Zwerger, Rev. Mod. Phys. 80, 885 (2008).

[10] B. Lanyon, C. Hempel, D. Nigg, M. Müller, R. Gerritsma, F. Zähringer, P. Schindler, J. Barreiro, M. Rambach, G. Kirchmair, M. Hennrich, P. Zoller, R. Blatt, and C. Roos, Science 334, 57 (2011).

[11] A. Eckardt, C. Weiss, and M. Holthaus, Phys. Rev. Lett. 95, 260404 (2005).

[12] A. Eckardt and M. Holthaus, Phys. Rev. Lett. 101, 245302 (2008).

[13] A. Lazarides, A. Das, and R. Moessner, Phys. Rev. Lett. 112, 150401 (2014).

[14] A. Lazarides, A. Das, and R. Moessner, Phys. Rev. E 90, 012110 (2014).

[15] E. Arimondo, D. Ciampini, A. Eckardt, M. Holthaus, and O. Morsch, Adv. At., Mol., Opt. Phys. 61, 515 (2012).

[16] J. Struck, C. Ölschläger, M. Weinberg, P. Hauke, J. Simonet, A. Eckardt, M. Lewenstein, K. Sengstock, and P. Windpassinger, Phys. Rev. Lett. 108, 225304 (2012).

[17] P. Hauke, O. Tieleman, A. Celi, C. Ölschläger, J. Simonet, J. Struck, M. Weinberg, P. Windpassinger, K. Sengstock, M. Lewenstein, and A. Eckardt, Phys. Rev. Lett. 109, 145301 (2012).

[18] N. H. Lindner, G. Refael, and V. Galitski, Nat. Phys. 7, 490 (2011).

[19] T. Prosen and E. Ilievski, Phys. Rev. Lett. 107, 060403 (2011).

[20] S. Mondal, D. Pekker, and K. Sengupta, Europhys. Lett. 100, 60007 (2012).

[21] V. M. Bastidas, C. Emary, G. Schaller, and T. Brandes, Phys. Rev. A 86, 063627 (2012).

[22] V. Mukherjee and A. Dutta, J. Stat. Mech. (2009) P05005.

[23] T. Nag, S. Roy, A. Dutta, and D. Sen, Phys. Rev. B 89, 165425 (2014).

[24] H. Lignier, C. Sias, D. Ciampini, Y. Singh, A. Zenesini, O. Morsch, and E. Arimondo, Phys. Rev. Lett. 99, 220403 (2007).

[25] A. Zenesini, H. Lignier, D. Ciampini, O. Morsch, and E. Arimondo, Phys. Rev. Lett. 102, 100403 (2009).

[26] Y.-A. Chen, S. Nascimbène, M. Aidelsburger, M. Atala, S. Trotzky, and I. Bloch, Phys. Rev. Lett. 107, 210405 (2011).

[27] J. Struck, C. Ölschläger, R. Le Targat, P. Soltan-Panahi, A. Eckardt, M. Lewenstein, P. Windpassinger, and K. Sengstock, Science 333, 996 (2011).
[28] U. Haeberlen, High-Resolution NMR in Solids: Selective Averaging, Vol. 1 (Academic Press, New York, 1976).

[29] J. Cavanagh, W. J. Fairbrother, A. G. Palmer III, and N. J. Skelton, Protein NMR Spectroscopy: Principles and Practice (Academic Press, San Diego, 1995).

[30] A. Das, Phys. Rev. B 82, 172402 (2010).

[31] T. W. B. Kibble, J. Phys. A: Math. Gen. 9, 1387 (1976).

[32] W. Zurek, Nature (London) 317, 505 (1985).

[33] B. Damski, Phys. Rev. Lett. 95, 035701 (2005).

[34] W. H. Zurek, U. Dorner, and P. Zoller, Phys. Rev. Lett. 95, 105701 (2005).

[35] J. Dziarmaga, Phys. Rev. Lett. 95, 245701 (2005).

[36] L. Landau and E. Lifshitz, Quantum Mechanics Non-relativistic Theory, Vol. 3 (Butterworth-Heinemann, Oxford, 1981).

[37] C. Zener, Proc. R. Soc. London A 137, 696 (1932).

[38] B. K. Chakrabarti and M. Acharyya, Rev. Mod. Phys. 71, 847 (1999).

[39] A. Messiah, Quantum Mechanics, Vol. II (English ed.; North Holland, Amsterdam, 1962).

[40] B. Damski and W. H. Zurek, Phys. Rev. A 73, 063405 (2006).

[41] S. Bhattacharyya, A. Das, and S. Dasgupta, Phys. Rev. B 86, 054410 (2012).

[42] A. Das and R. Moessner, arXiv:1208.0217.

[43] M. Bukov, L. D’ Alessio, and A. Polkovnikov, arXiv:1407.4803.

[44] S. Suzuki, J.-i. Inoue, and B. K. Chakrabarti, Quantum Ising Phases and Transitions in Transverse Ising Models (Springer, Heidelberg, 2013).

[45] D. H. Dunlap and V. M. Kenkre, Phys. Rev. B 34, 3625 (1986).

[46] F. Grossmann, T. Dittrich, P. Jung, and P. Hänggi, Phys. Rev. Lett. 67, 516 (1991).

[47] J. Zhang, X. Peng, N. Rajendran, and D. Suter, Phys. Rev. Lett. 100, 100501 (2008).

[48] J. Zhang, F. M. Cucchietti, C. M. Chandrashekar, M. Laforest, C. A. Ryan, M. Ditty, A. Hubbard, J. K. Gamble, and R. Laflamme, Phys. Rev. A 79, 012305 (2009).

[49] E. Lieb, T. Schultz, and D. Mattis, Ann. Phys. 16, 407 (1961).

[50] D. C. Mattis, The Theory of Magnetism Made Simple: An Introduction to Physical Concepts and to Some Useful Mathematical Methods (World Scientific, Singapore, 2006).

[51] B. Damski and M. M. Rams, J. Phys. A: Math. Theor. 47, 025303 (2014).

[52] See Supplemental Material at http://link.aps.org/supplemental/ 10.1103/PhysRevB.90.174407 for details on NMR states, decoherence and inverse decay techniques.

[53] N. Khaneja, T. Reiss, C. Kehlet, T. Schulte-Herbrüggen, and S. J. Glaser, J. Magn. Reson. 172, 296 (2005).

[54] J. Kowalewski and L. Maler, Nuclear Spin Relaxation in Liquids: Theory, Experiments, and Applications, Vol. 2 (Taylor \& Francis, London, 2006).

[55] V. Mukherjee, A. Dutta, and D. Sen, Phys. Rev. B 77, 214427 (2008).

[56] To get this form, we have added a term $(\delta \cos k) \mathcal{I}_{k}$ to the Hamiltonian, where $\mathcal{I}_{k}$ is the $2 \times 2$ identity matrix in the representation of Eq. (8), which contributes nothing but an additional overall phase to the wave function. 